\title{
Mass Distribution Campaign of Long-Lasting Insecticidal Nets in the Democratic Republic of Congo from 2018 to 2021 in the Context of the Emergence of COVID-19: Results and Lessons Learned
}

\author{
Joris Losimba Likwela ${ }^{1,2}$, Adrien N'siala Kumbi ${ }^{1}$, Michèle Luntadila Kiamenga ${ }^{1}$, \\ Mireille Lusiense Zena ${ }^{1}$, Daniela Mundele Iris ${ }^{1}$, Simon-Pierre Kazadi Mutuba ${ }^{1}$, \\ Eric Mukomena Sompwe ${ }^{3,4}$, Patrick Kanku-Ka-Lukusa', Albert Kalonji Ntumba1, \\ Ghislain Makhan Yav5, Didier Gasigwa Baneti' ${ }^{5}$ Eric Tsasa Mbuku6, \\ Charlotte Ndolerire Isingoma ${ }^{6}$, Muriel Nzazi Nsambu7 ${ }^{7}$, Philippe Lukanu Ngwala1,8 \\ ${ }^{1}$ SANRU Asbl, Kinshasa, Democratic Republic of the Congo \\ ${ }^{2}$ Public Health Department, Faculty of Medicine and Pharmacy, University of Kisangani, Kisangani, \\ Democratic Republic of the Congo \\ ${ }^{3}$ National Malaria Control Program, Kinshasa, Democratic Republic of the Congo \\ ${ }^{4}$ School of Public Health, University of Lubumbashi, Lubumbashi, Democratic Republic of the Congo \\ ${ }^{5}$ Financial Support and Management Unit, Ministry of Public Health, Prevention and Hygiene, Kinshasa, \\ Democratic Republic of the Congo \\ ${ }^{6}$ Interchuch Medical Assistance World Heath, Kinshasa, Democratic Republic of the Congo \\ ${ }^{7}$ World Health Organisation, Kinshasa Sub-Office, Democratic Republic of the Congo \\ ${ }^{8}$ Department of Family Medicine et Primary Health Care, Protestant University of Congo, Kinshasa, \\ Democratic Republic of the Congo \\ Email: jorislikwela@gmail.com
}

How to cite this paper: Likwela, J.L., Kumbi, A.N., Kiamenga, M.L., Zena, M.L., Iris, D.M., Mutuba, S.-P.K., Sompwe, E.M., Kanku-KaLukusa, P., Ntumba, A.K., Yav, G.M., Baneti, D.G., Mbuku, E.T., Isingoma, C.N., Nsambu, M.N. and Ngwala, P.L. (2022) Mass Distribution Campaign of Long-Lasting Insecticidal Nets in the Democratic Republic of Congo from 2018 to 2021 in the Context of the Emergence of COVID-19: Results and Lessons Learned. Journal of Biosciences and Medicines, 10, 136-159.

https://doi.org/10.4236/jbm.2022.102013

\begin{abstract}
Introduction: The DRC had planned 23 mass distribution campaigns distribution of long-lasting insecticidal nets (LLIN) for the years 2018-2020, the implementation of which spanned from 2018 to July 2021. This article reviews the campaign's planning process, the results, challenges, and lessons learned. Methods: A descriptive method was used to postpone the planning and implementation process according to the National Malaria Control Program (NMCP) standards adapted to the COVID-19 context. The changes and adaptations implemented as well as the challenges encountered are described. Results: Between January 2018 to June 2021, 23 LLIN mass distribution campaigns were organized in the DRC with the financial support of The Global Fund to Fight against Tuberculosis, AIDS and Malaria (GFTAM) and Against
\end{abstract}


Received: January 9, 2022

Accepted: February 25, 2022

Published: February 28, 2022

Copyright (C) 2022 by author(s) and Scientific Research Publishing Inc. This work is licensed under the Creative Commons Attribution International License (CC BY 4.0).

http://creativecommons.org/licenses/by/4.0/
Malaria Foundation (AMF) allowing the distribution of 55,273,473 LLINs to $19,048,372$ households at risk of malaria transmission with an average of 2.9 LLINs per household. The enumerated population $(111,081,191)$ exceeded $7 \%$ of the micro plans projected population $(102,790,391)$ while the number of households enumerated $(19,311,629)$ was $3 \%$ lower compared to the micro planning projection households $(19,862,417)$. Compared to a reported household coverage of $96 \%$ of households achieved over the expected households, the independent monitoring carried out revealed $91 \%$ of households served in the intervention areas. The main reasons for not reaching households mentioned by the respondents were absence at the time of distribution (26\%) followed by the loss of vouchers (16\%). Several communication channels were used among which, community workers were the most frequently mentioned (63.1\%), followed by radios (18.5\%) and churches (12.4\%). Conclusion: Good planning, effective coordination of stakeholders, and revision of the implementing campaigns methods following the COVID-19 were factors in the success of this campaign. An effort to respect the schedules for renewing LLINs in households, coupled with good continuous distribution, is necessary to maintain the gains and hope for an impact in terms of morbidity and mortality reduction of malaria.

\section{Keywords}

Vector Control, LLIN Campaigns, COVID-19, Lessons, Democratic Republic of Congo

\section{Introduction}

During the development of the global technical strategy for malaria 2016-2030 (GTS 2016-2030) and the call for RBM Partnership to end malaria in "Action and Investment to Defeat Malaria 2016-2030" (AIM 2016-2030), the World Health Organization (WHO) estimated a 212 million malaria cases and 429,000 deaths due to malaria worldwide in 2015 [1] [2] [3]. This represented a reduction of $22 \%$ of cases and $50 \%$ of deaths compared to the situation in 2000 [3]. This impressive progress was due to the development in the 1990s of new disease control tools, including LLINs, rapid diagnostic tests, and more effective drugs combinations. The 2000s marked the increasing of investments in the fight against malaria, the creation of new funding mechanisms, notably the Global Fund to Fight Tuberculosis, AIDS, and Malaria (GFTAM), and the United States President's Malaria Initiative (PMI), which allowed a large-scale deployment of these new tools [4].

Between 2015 and 2019, malaria incidence globally decreased by only $2 \%$, reflecting a sharp slowdown since 2015 [4]. To revive the momentum for progress, the WHO and the Roll Back Malaria (RBM) partnership, initiated in 2018, the "from a high burden to a strong impact" approach (HBHI) for 11 countries which account for nearly $70 \%$ of malaria cases and deaths in the world, with 10 in 
sub-Saharan Africa, including the Democratic Republic of Congo (DRC) [4] [5]. Since the DRC NMCP targeted ten provinces which concentrated $64 \%$ of malaria cases in 2019 to intensify malaria fighting [6].

GTS 2016-2030, AIM 2016-2030, and HBHI converge on the need for universal coverage of at-risk populations of malaria through key interventions, including the use of LLINs, requiring an investment to achieve the malaria morbidity and mortality reduction targets set at $90 \%$ in 2030 compared to the situation in 2015 [1] [2] [5].

The use of LLINs has increased in sub-Saharan Africa (SSA) in the past decade leading to a significant drop in malaria morbidity and mortality in Africa despite the emergence of vector resistance to insecticides [7] [8] [9] [10]. The dysfunctions of health systems due to COVID-19 have led to cancellations and postponements of LLIN distribution campaigns, threatening to reverse the progress made [11] [12] [13].

The promotion of LLINs use is the primary vector control strategy promoted by the DRC NMCP [6]. Between 2007 and 2014, DRC experienced a drastic increase in the possession and use of LLINs following significant investments, mainly from GFTAM and PMI, supporting the DRC NMCP [14] [15]. Secondary analyzes of data from the Demographic and Health Survey (DHS) 2013-2014 showed that the use of LLINs was significantly associated with a reduction of malaria prevalence by up to $44 \%$ and in malaria mortality by $41 \%$ in children under 5 in the DRC [16] [17] [18].

Mass distribution campaigns are the best way to rapidly increase LLIN coverage of at-risk populations to achieve impact [19]. In the DRC during 2017, out of the eight provinces planned for LLIN mass distribution campaigns, only one had been fully distributed mosquito nets and another partially [20]. At the same time, the NMCP reported an increase in malaria cases from 12,186,639 cases in 2016 to $15,272,767$ cases in 2017 and 18,208,440 in 2018 [20] [21] [22]. Therefore, it was important for the NMCP to speed up the organization of campaigns in the next years to catch up and align with the WHO's call for the intensification of the fight and its focus on HBHI countries, including the DRC.

The objective of this study was to review the planning process of LLIN mass distribution campaigns for the years 2018-2020, the implementation of which spanned from 2018 to 2021, and to report obtained results, challenges faced, and lessons learned to guide future distribution campaigns in DRC and other African countries.

\section{Methods}

\subsection{Description of the LLIN Mass Campaign Organization Cycle in the DRC}

The DRC had started the mass distribution of LLINs with campaigns associated with vaccination against Measles, polio, vitamin A supplementation as well as deworming with Mebendazole targeting children under 6 to 36 months in the 
province of Bas-Congo in 2006, then children aged 0 to 5 in the provinces of South Kivu in 2007 and Equateur in 2008.

The first LLIN distribution campaigns started in 2009 in the Orientale and Maniema provinces. The gradual mobilization of resources made it possible to complete the 1st distribution cycle throughout the country only in 2012. With the 1st round of the GFTAM New Funding Model (NFM1) from 2014-2017 followed by the NFM2 from 2018-2020 coupled with the financing of PMI that the DRC has managed to stabilize a distribution cycle covering the whole country in 3 years and renewing with an interval of 3 years for each province. The Against Malaria Foundation (AMF) contribution in purchasing LLINs from 2019, continuing to cover 9 of the 26 provinces, was decisive in setting these targets within a constraint of limited resources.

From 2018 to 2020, all 26 provinces were planned for mass LLINs distribution campaigns with financial support from the GFTAM (21 provinces) or PMI (5 Provinces) (Figure 1) [6].

For the 21 provinces benefiting from GFTAM funding, funds for 15 provinces went through two principal recipients, namely Primary Health Care in Rural Areas/Non-profit organization (SANRU Asbl) for logistics, communication, and

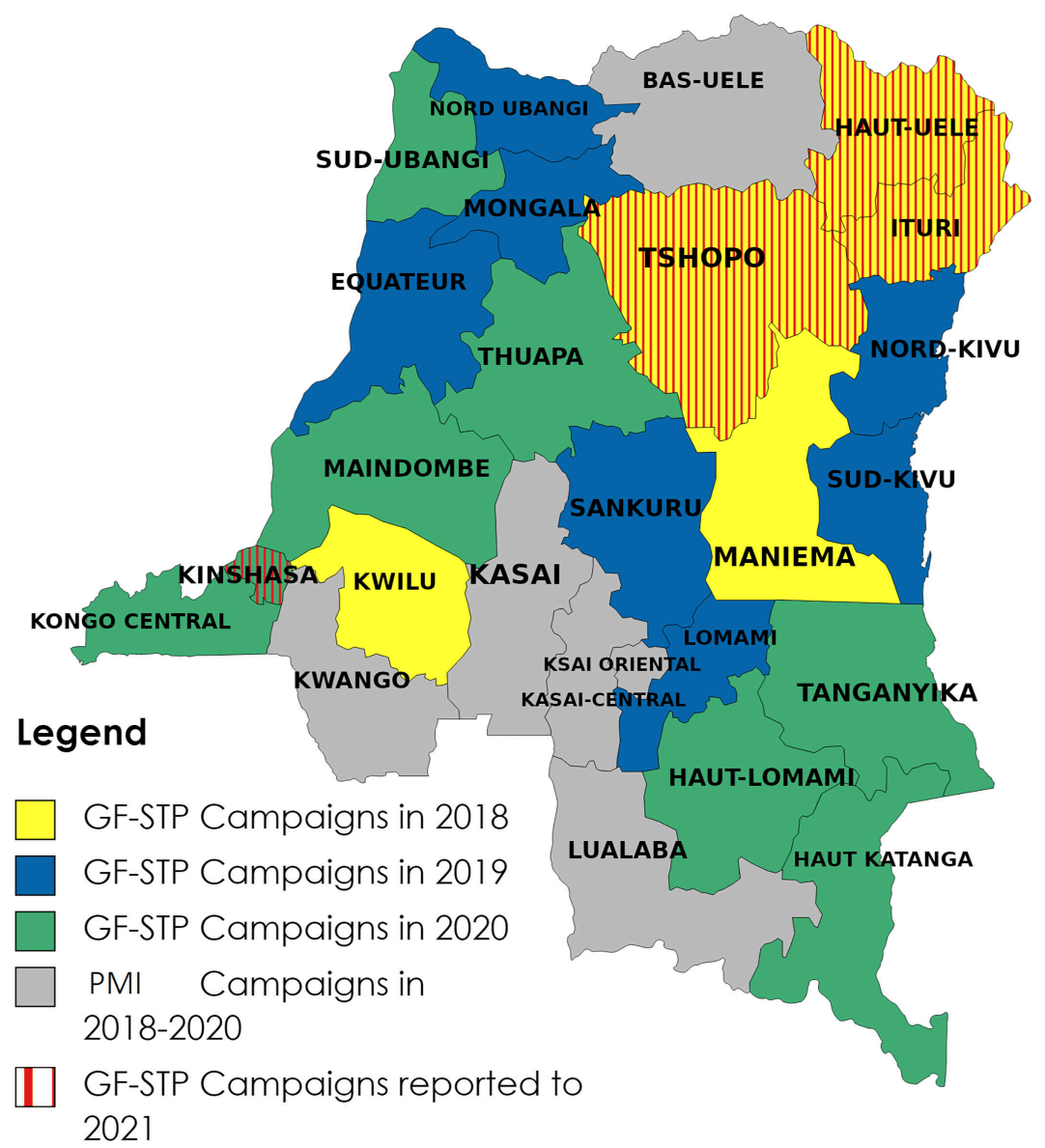

Figure 1. LLIN distribution campaigns planned for the 26 provinces of the DRC between 2018 and 2020 . 
monitoring aspects and the Ministry of Health Financial Management Support Unit (FMSU) for planning activities, training, LLINs transport from the central offices of the Health Zone (HZ) to the health area (HA) and from there to villages, household count, distribution of LLINs to households and independent monitoring. For the other six provinces, SANRU was the principal recipient but relied on Interchurch Medical Assistance world heath (IMA world health) as a sub-recipient which relayed the support to the HZ for all the activities supported by GFTAM in the other provinces.

It should be noted that, among the 5 LLINs mass distribution campaigns with GFTAM funding planned in 2017, four were carried out in 2018, in the provinces of Ituri, Haut Uélé, Maniema, and Tshopo.

This study focuses on the campaign's funding the GFTAM, including the four campaigns postponed from 2017 to 2018 and the 21 campaigns planned for 2018-2021 with co-financing from AMF to purchase the LLINs from 2019 for 9 out of 21 provinces.

\subsection{DRC Epidemiological Profile}

The entire population is at risk of malaria infection, which is the leading cause of morbidity and mortality. In 2019, the reported incidence of the disease was 327 cases per 100,000 inhabitants, with in-hospital mortality attributed to malaria of 40 deaths per 100,000 inhabitants [22]. Almost the entire population (97\%) lives in areas with stable transmission, while a small part of the population (3\%) living in the eastern highland area faces seasonal malaria transmission with a risk of malaria outbreaks [23].

Available data show several vectors existing in the country, being mainly Anopheles gambiae (92\%). Plasmodium falciparum is the predominant parasitic species (95\%), with Plasmodium ovale and Plasmodium malariae more rarely encountered [23]. Although Plasmodium vivax has been assumed to be absent from sub-Saharan Africa because of the protective mutation conferring the Duffy-negative phenotype, recent evidence has suggested that $P$. vivax infections have been identified in DRC [24] [25].

Anopheles gambiae, the main malaria vector encountered in the country, is resistant to permethrin and a lesser extent, to deltamethrin. Entomological survey data show that, although the presence of the kdr-1014F mutation was very common, the increased mortality of Anopheles gambiae after exposure to piperonyl butoxide $(\mathrm{PBO})$ indicates that this resistance is partly due to metabolic mechanisms [26].

\subsection{Campaign Planning and Implementation Process}

Prior to the COVID-19 pandemic, the planning process of LLIN distribution campaigns in the DRC consisted of 4 key steps: macroplanning, household enumeration, microplanning, and then the distribution of LLINs to households (Figure 2) [27]. Following the occurrence of the COVID-19 pandemic, to reduce the transmission risk, the NMCP decided to link the household enumeration to 


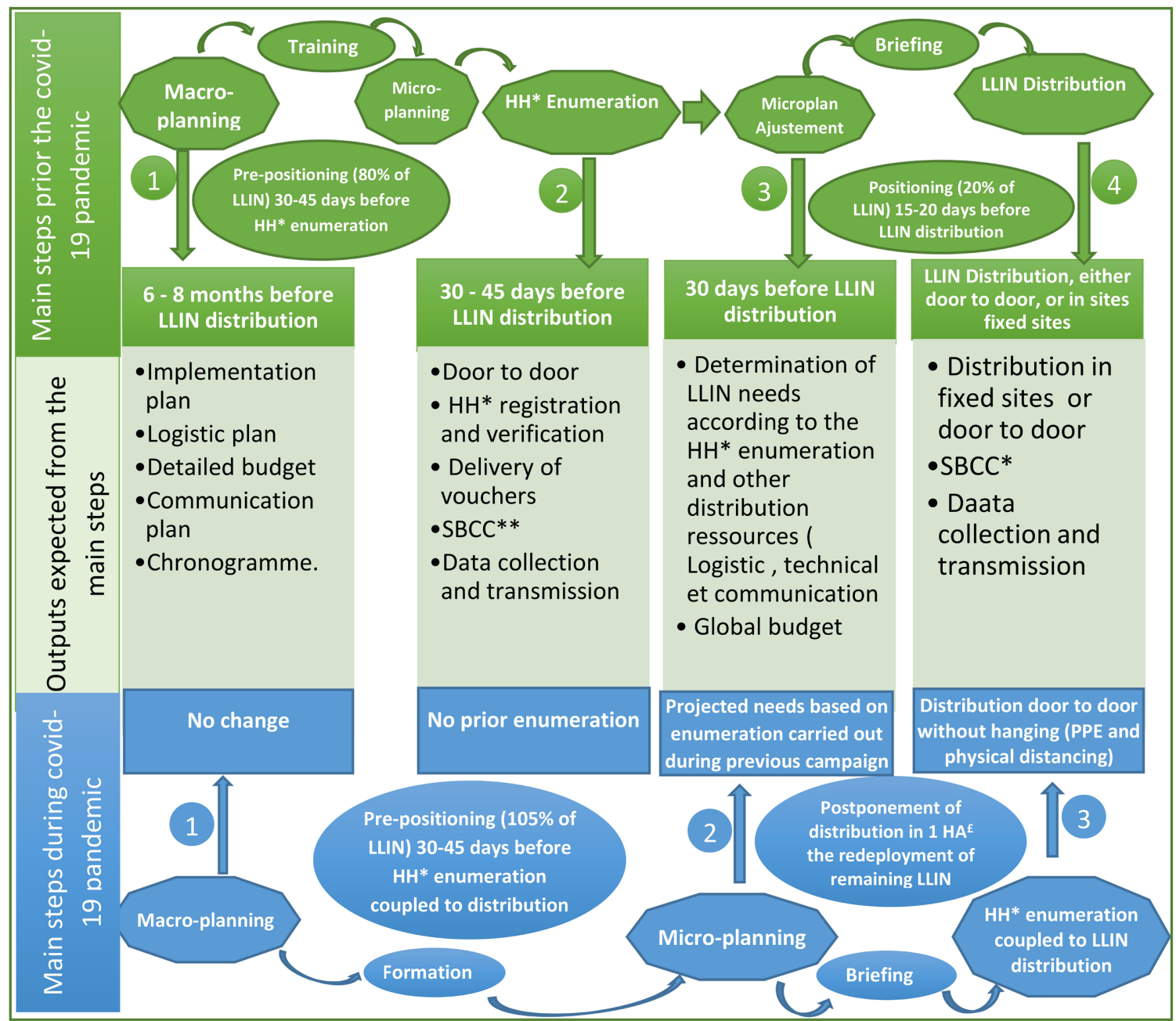

$\mathrm{HH}^{*}$ : Household ; SBCC** : Social and Behavior Change Communication ; HA ${ }^{f}$ : Health area.

Figure 2. Key stages in the planning and implementation process of mass LLIN campaigns in the DRC and the changes induced by COVID-19.

LLIN distribution to avoid double exposure of household's distribution teams, particularly in the context of fixed-site distributions, which caused population surge that was difficult to control [28].

Macroplanning had to be done early, 6 to 12 months before the distribution, depending on whether the provincial campaigns were scheduled for the year's 2nd, 3rd, or 4th quarter. Thus, this process was planned for the fourth quarter preceding the year of implementation. Its objective was to define the implementation strategy, determine the needs in terms of LLIN quantity, human and material resources required and estimate the estimated overall cost of the campaign, based on the population of the most recent population count.

The macro-planning development workshop was firstly held at the provincial level for five days with the core team of the provincial health divisions (CTPHD) and the NMCP Provincial Coordination. Then, the delegates from all targeted provinces were invited to a national workshop for annual macro-planning consolidation. The deliverables expected were: an implementation plan with a 
provisional budget highlighting a rigorous quantification of the needs to achieve universal coverage of households with LLINs, a detailed timetable, a logistic plan with a deployment plan taking into account the provinces realities, and a communication plan anticipating the bottlenecks encountered during the previous campaigns.

During the years 2018 and 2019, about two months before the households' enumeration ( 3 to 4 and a half month before LLIN distribution), successive training workshops in the management of LLIN mass distribution campaign were organized for five days to strengthen the capacity of the CT-PHD and core teams of the HZ (CT-HZ) followed by those of the titular nurses (TN) of the health areas (HA) and the chairpersons of the development committees of the HA (CDC-HA) (3 days). The TN and CDC-HA training workshop ended with the development of the first format of HA microplans that will be consolidated into HZ microplans. At the end of the chain, the TNs briefed the community health workers (CHW) on household enumeration procedures (1 day). In 2020, following the occurrence of the COVID-19 pandemic, physical distancing led to the limitation of the number of participants per room (with a consequent increase in the number of rooms, the number of facilitators, and support staff), the provision of masks and bottles of hydro-alcoholic gels had been put in place to reduce the risk of transmission of this disease.

Based on the microplans, Provinces organized the enumeration of households at the HZ level. In the provinces where the "fixed-site" or "door-to-door" distribution methods were selected, each CHW recorded 25 households per day in rural areas or 30 households per day in urban areas. In those where distribution was to be carried out by community participation mechanisms, each CHW had to record 50 households per day, regardless of the environment. After the households were registered, a voucher was given to the heads of household to serve 30 to 45 days later, as an access ticket to the LLINs required according to their household size. In 2020, CHW and their supervisors were also equipped with masks and bottles of hydro-alcoholic to reduce COVID-19 risk of transmission.

After workshops to validate the enumeration data, the CT-HZ adjusted the microplan to obtain the final data to quantify the needs and determine the resources required for the LLINs distribution to households. As for training, in 2020, during data validation workshops, physical distancing measures restricting the number of participants per room (with as a consequence increased number of rooms, facilitators, and staff), the endowment of masks and bottles of hydro-alcoholic gels had been put in place to reduce the risk of transmission of COVID-19.

To be reassured of the quality of the service provided at the time of LLIN distribution, a series of one-day briefings were organized to refresh the actors on the modalities of LLIN distribution. The NMCP team led the CT-PHD briefing. The CT-PHD, in turn, briefed the HZ team, and the latter briefed the TN and CDC-HA. At the end of the chain, the TN briefs the CHW who subsequently 
become the distribution teams for LLINs to households. These briefings were given, in 2020, in compliance with the measures above to prevent the transmission of COVID-19.

About two months after the enumeration, the LLIN distribution to households was organized according to one of the three methods below:

- Either a "fixed-site" distribution: the heads of households with a voucher received during the enumeration went to a site planned to distribute LLINs to households in 4 days. Each site maintained by $4 \mathrm{CHW}$ (1 law enforcement officer, one distributor, one pointer, and one mobilizer) had to distribute LLINs every day to 200 households in rural areas or 300 households in urban areas.

- Either a "door-to-door" distribution: teams of 2 CHW (1 LLIN hanger and one recorder) had to serve 20 households per day in rural areas or 30 households per day in urban areas for five days.

- Either a "community" distribution: the distribution was organized by the community animation units (CAU) by unique village/single avenue or villages/avenues grouped according to the size of the villages. This distribution was done in 3 days in rural areas or four days in urban areas.

After the occurrence of COVID-19 outbreak in the 1st quarter of 2020, the NMCP decided, with the support of donors and PTFs, in addition to coupling the household enumeration to the distribution, to switch all distributions to the "door to door" method to avoid groupings of the population that could increase the risk of disease transmission.

\subsection{Supply and Inventory Management}

The criteria retained by the NMCP to choose the types of LLINs to be distributed in the country were: 1) the manufacturer, the manufacturing site, and the LLIN must be prequalified by the WHO;2) the manufacturer had to present a certificate from the International Organization for Standardization (ISO); 3) in case of supplier was other than the manufacturer, he had to present a letter of commitment; 4) the product must have obtained a Marketing Authorization (AMM) granted by the MOH Pharmacy and Medicines Department (PMD).

The forecasted quantification was based on the population estimated based on data from the previous enumeration to which the natural increase rate of $2.9 \%$ provided by the National Statistical Institute (INS) was applied [29]. Based on the WHO recommendation, the number of LLINs to ensure universal coverage was then obtained by using the formula [30]:

$$
\text { Quantity of LLINs required }=(\text { Estimated population }) / 1.8
$$

About 9 to 12 months before the planned distribution date, the lessors placed the orders and signed a contract with a procurement and transit agency. MOH/NMCP support agencies for implementing campaigns (SANRU for GFTAM, UNICEF, or CHEMONICS for PMI) were responsible for monitoring orders and receiving LLINs at the entry points of the country at least 3 - 4 months before distribution. 
These agencies then ensured the deployment of the LLINs to the HZs in the beneficiary provinces at the rate of $80 \%, 30$ to 45 days before the distribution, then the $20 \%$ temporarily kept in a provincial warehouse were completed according to the readjusted data of the microplans after household enumeration. The CT-HZ organized the deployment of LLINs from the HZ warehouses to the HA and distribution sites, respectively, "in fixed sites" or villages for "door-to-door" or "community" distributions at least seven days before the launch of the distribution.

With the occurrence of the COVID-19 outbreak, to the initial quantification, $5 \%$ of contingency stock was added to anticipate any possible stock-out following the uncertainty about populations that may result from their movements since the last enumeration. The risk involved was to see a slightly larger remainder after the distribution. A second adjustment lever was to launch the distribution in all HA of an HZ except the one that hosts the Central Office of the $\mathrm{HZ}$ (COHZ), which had to be shifted and whose LLINs could serve as additional stock to be used in the event of stock out. At the end of the distribution, a stock reconciliation should allow redeployments between $\mathrm{HA}$ or between $\mathrm{HZ}$ to finalize the distribution in the "shifted" HA.

\subsection{Campaign Coordination}

Campaign coordination was organized at all levels of the health pyramid:

- At the national level: a National Technical Committee (NTC) met weekly to monitor activities implementation, provide feedback to the provinces level, and prepare key elements decision-making during the meetings of NTC.

- At the provincial level: a Provincial Coordination Committee (PCC) ensured the follow-up of the smooth running of the campaign in the HZ. A total of 10 meetings were to be held at the rate of two during the preparatory phase, one daily during the distribution and two after the distribution for a rapid evaluation of the campaign results before the final validation workshop, which should be held two weeks after the end of the campaign.

At the HZ level: the Local Coordination Committees (LCC) ensured the close monitoring of the smooth running of the campaign in the HA through meetings held at the same frequency as that of the PCC with an additional session on the day of the catch-up of unreached households.

\subsection{Communication to Support LLIN Distribution Campaign}

Communication was considered to be a significant lever for the success of campaigns. The planning of the related activities went through a community diagnosis organized around 4 to 6 months before the distribution of the LLIN, followed by a workshop to readjust the communication plan developed during the macro-planning phase.

The strategies used to support the LLIN distribution campaigns were: 1) advocacy targeting community and political leaders to obtain their ownership of 
the activities and their commitment to mobilize the populations and help remove any bottlenecks; 2) lobbying using civil society organizations (CSO) and community-based organizations (CBOs) to ensure "citizen" control contributing to good governance in the management of resources allocated to the campaign and to community mobilization; 3) social mobilization aimed at putting into action all the societal forces and operational networks in the communities, in particular schools and universities, the national police, the armed forces of the DRC, non-governmental organizations (NGOs), associative movements, communuty based organization $(\mathrm{CBO})$ with particular emphasis on partnership with religious denominations, women's associations and youth movements as well as the media in raising public awareness; 4) mass communication using channels with a large audience such as: radios and televisions; 5) non-media communication such as billboards, posters, banners, leaflets...; 6) proximity communication using CHW either to animate educational talks, to make home visits or with voice throwers passing village by village and avenue by avenue to announce the key dates of the campaign (enumeration, distribution), communicate on the importance of the LLIN and its good use...; 7) solemn launching ceremonies constituting an opportunity to ensure the visibility of the launch of the distribution translated through distinctive signs ( $\mathrm{T}$-shirts, posters, leaflets during the LLIN presentation) and cultural productions attracting the population and allowing to mark it by messages conveyed by artists locally very appreciated and by the politico-administrative authorities.

\subsection{Monitoring and Evaluation of the Campaign}

In 2018, the enumeration and distribution data began at the community level by filling out vouchers and registers. From 2019, in the provinces supported by SANRU Asbl with IMA World Health as sub-recipient, campaign data started to be collected using tools based on Open Data Kit (ODK) developed by IMA world health using smartphones. Then, with the contribution of the AMF in the purchase of LLINs, there was a scaling up of the digitization of data collection for all campaigns through the development of digitization tools by SANRU Asbl, also based on ODK.

In any case, two days after the end of the distribution, all HA had to complete collection and be validated for transmission to the CT-HZ. One week after the distribution, the CT-HZ was to centralize and validate data before the provincial data validation workshop.

In addition to the data thus collected under the coordination of the CT-HZ with the support of the CT-PHD, independent surveys were carried out either by the national coordination of networks of civil society organizations (NCNCSO) working in the field of health, by the provincial offices of WHO or by a research institution.

WHO Provincial Offices or research institutions used the "lot quality assessment sampling" (LQAS) method for sampling households to be surveyed: 1) 
random selection of $2 / 3$ of $\mathrm{HZ}$ using the random number table; 2) for each $\mathrm{HZ}$ retained, a random selection of 6 localities/villages/street by the same method; 3 ) in the selected locality/village/street, the total number of households was divided by 10 (10 being the number of households to be surveyed) to obtain the sampling interval $\mathrm{k}$; 4) the 1 st household to be surveyed was selected randomly between 1 and $\mathrm{k}$ using a random number table after a plot survey assigning numbers to all the plots of the locality/villages/street; 5) the following households were chosen after a step of $\mathrm{k}$ households starting to the right of the 1st household until a total of 10 households per locality/village/street for 5 to 6 days. Data collection was carried out using a structured questionnaire with closed and open-ended questions, administered through a personal interview by investigators trained and supervised daily.

In provinces where neither the WHO nor a research institution was involved, the data from was monitoring carried out by NCNCSO were used. In these cases, multi-stage sampling was applied: 1) selection of up to $15 \mathrm{HZs}$ using urn method without replacement (for provinces with $\leq 15 \mathrm{HZ}$, all HZs were included); 2) in each selected HZ, $2 \mathrm{HA}$ or $4 \mathrm{HA}$ were randomly selected by the same method depending on whether the province had $\leq 15 \mathrm{ZS}$ or $>15 \mathrm{ZS}$; 3) in each HA, the village hosting the Health Center was selected for logistical reasons; 4) the starting point was randomly determined between 1 and 3 by considering the household of the head of the locality as household $\mathrm{N}^{\circ} 1$ and then following the direction determined by the pen thrown in the air; 5) the following households were identified by adding a sampling step of 3 households until the required number was reached. The sample size was set at $1.5 \%$ of households in the province distributed evenly across the selected HZs and HA. In the provinces where WHO and research institutions had worked, data collection was carried out using a structured questionnaire with closed and open questions, administered through a personal interview by investigators trained and supervised daily.

Among the indicators used for campaign evaluation, the following are presented in this article:

- The gap between enumerated population and projected population $=($ Enumerated population-projected population $) /($ Projected population $) \times 100$;

- The gap between enumerated households and projected households $=(\mathrm{Enu}-$ merated households-projected households)/(Projected households) $\times 100$;

- The gap between households served and enumerated households = (Households served-enumerated households)/(Enumerated households) $\times 100$;

- The proportion of households served $=$ ( households served $) /($ projected households) $\times 100$ (This is the proportion of households served as reported by the HZ team, local distribution coordination structures);

- Household coverage $=($ Lhouseholds served $) /($ households surveyed $) \times 100$ (This is the proportion of households served as collected by independent monitoring carried out either by the provincial office of the WHO, by a research institution, or by the CNRSC);

- The Proportion of LLIN distributed $=\left(\sum L L I N\right.$ distributed $) /\left(\sum M I L D\right.$ required $)$ 
$\times 100$;

- The gap between the quantity of LLIN planned and the amount of LLIN received at the country's entry doors = Quantity of LLIN received at the entry door - Quantity of LLIN required;

- The LLIN balance at the entry point = Quantity of LLIN shipped to HZ Quantity of LLIN received at the entry point;

- The gap between the quantity of LLIN received in the HZs, and the amount of LLIN shipped to the HZs = Quantity of LLIN received in the HZs - Quantity of LLIN sent to the HZs;

- The Theoretical balance of LLINs in HZs = Quantity received in HZs Quantity of LLINs distributed to households;

- The Certified LLIN balance in HZs = Physical balance after contradictory inventory between $\mathrm{HZ}$ team and a SANRU Sub-Recipient agent;

- The Loss of LLINs in HZs = Theoretical balance of LLINs in HZs-certified balance of LLINs in HZs.

- The Loss rate $=($ Number of LLINs lost in HZs $) /($ Quantity received in HZs $) \times$ 100 ;

- The Proportion of different sources of information on LLINs cited by respondents $=\left(\sum\right.$ respondents who cited the source $) /\left(\sum\right.$ People surveyed $) \times 100$;

- The Proportion of unserved households $=\left(\sum\right.$ unserved households $) /\left(\sum\right.$ Households surveyed) $\times 100$;

- The Proportion of different causes of non-reaching households during LLIN distributions $=\left(\sum\right.$ number of respondents who cited a cause $) /\left(\sum\right.$ Number of people surveyed) $\times 100$.

\section{Results}

During the 23 LLIN distribution campaigns organized in 20 provinces, a total of 111,081,191 people were enumerated in 19,311,629 households (Table 1).

There was an average increase of $7 \%$ in the enumerated population compared to the projected population in microplans and a $3 \%$ reduction in enumerated households compared to the projected microplanning number of households (Table 1). Before COVID-19, distribution was preceded by enumeration, and the proportion of unserved households compared to enumerated households was $-1 \%$. The average number of people living in households was 5.8 people, ranging from 4.4 in Haut Uélé in 2018 to 6.8 in Kinshasa in 2021.

A total of 19,048,372 households were served during the 23 campaigns organized. The proportion of households served was $96 \%$ compared to projections made at microplanning, with variations ranging from 59\% in MONGALA province in 2019 to $118 \%$ in Haut Uélé province in 2018 (Figure 3). Independent monitoring carried out 2 to 7 days after distribution showed that $91.3 \%$ of the households surveyed had been served with LLINs with variations ranging from $75 \%$ in the province of Haut-Uélé in 2021 to $100 \%$ in the province North Ubangi and Lomami in 2019 (Figure 3). 
Table 1. Population and households enumerated compared to projections from microplans.

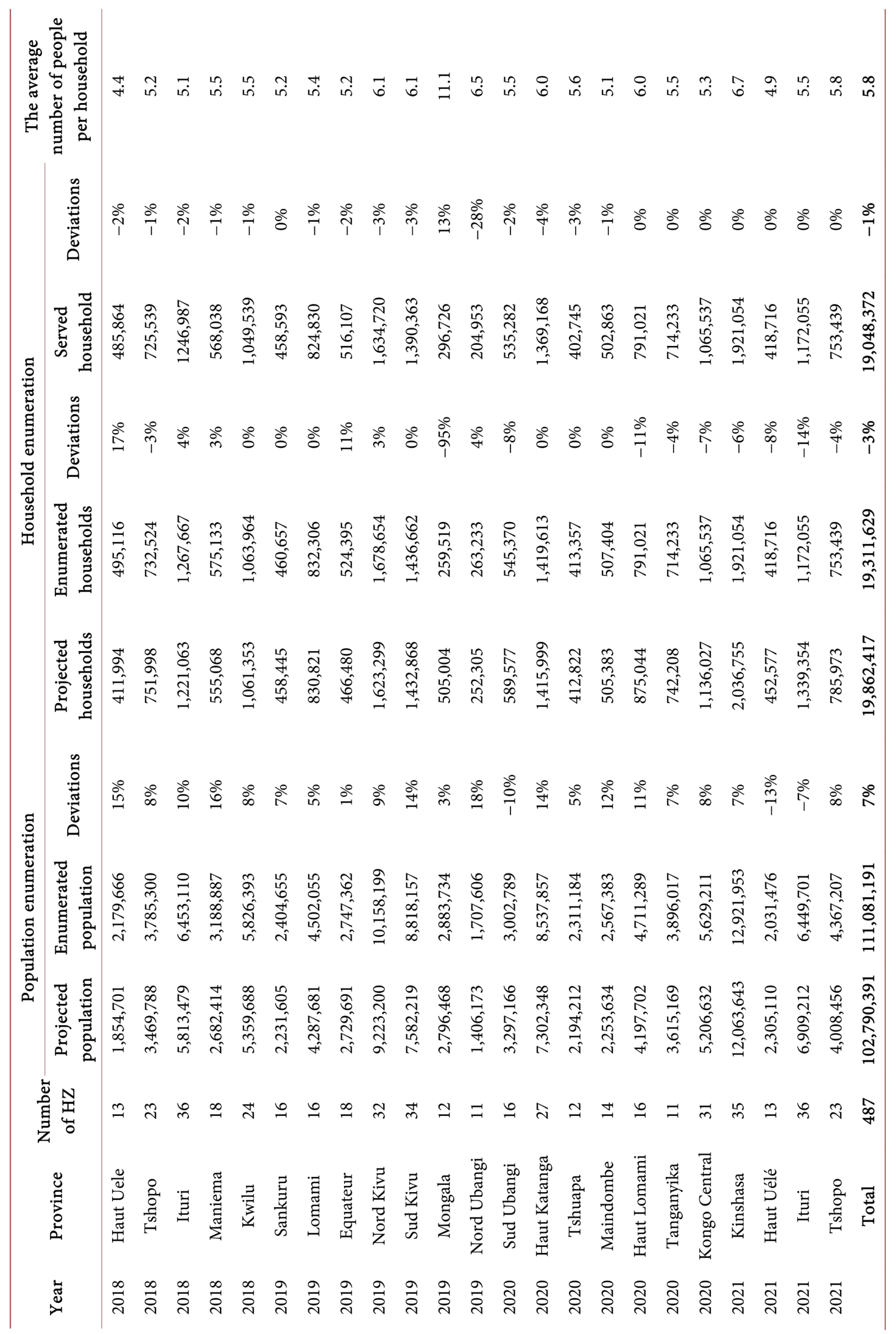




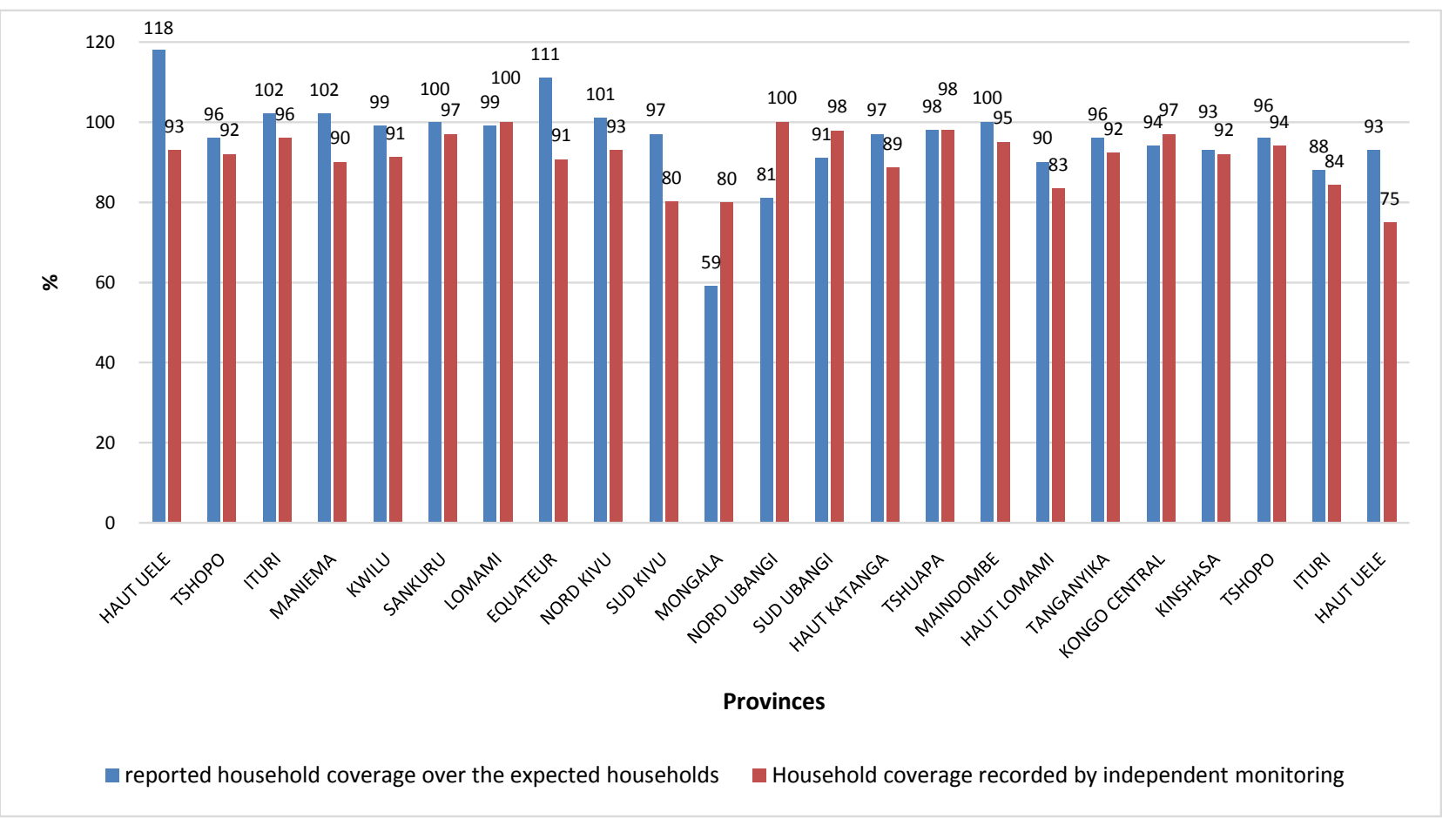

Figure 3. The proportion of households served compared to projected households vs. coverage of surveyed households.

Table 2 shows the results of various monitoring surveys, which revealed an average of $8.7 \%$ of households not served with LLINs. The main reasons mentioned by respondents were absence at the time of distribution (26\%), loss of vouchers $(16 \%)$. However, considering each province individually, certain reasons had a significant role among the reasons that did not allow households to access LLINs, such as stockouts in the Tshopo (34\%) in 2018, households not visited in the door-to-door distribution in the province of Tshuapa (21\%) in 2020 and Kinshasa (21\%) in 2021.

Overall, the proportion of LLINs distributed to households compared to the planned quantities was $98 \%$, with a variation from $90 \%$ in Nord Kivu to $110 \%$ in Kongo Central (Figure 4).

Out of a total of 56,267,554 planned LLINs, overall, we had received 1,844,163 additional LLINs (i.e. 3.3\%), particularly for some of the provinces that distributed in 2020 and those of 2021. Of the 58,111,717 LLINs received at the country entry point, 57,056,293 were sent to the HZ, and a difference of 1,055,424 LLINs was kept at the entry point. 55,581,961 LLINs were distributed to households during the 23 campaigns organized (Table 3). The proportion of LLINs distributed compared to planned targets was $98.8 \%$. The reception rate at the HZ level was $99.9 \%$. Finally, after the distribution of LLINs to households, the overall loss rate was $0.31 \%$, with a variation ranging from $0.00 \%$ in Katanga to $2.78 \%$ in Mongala province.

Table 4 shows that, overall, the most common source of information cited by respondents was RECOs/mobilizers/town criers (63.1\%), followed by radios (18.5\%) and then churches (12.4\%). 
Table 2. Reasons for not reaching households.

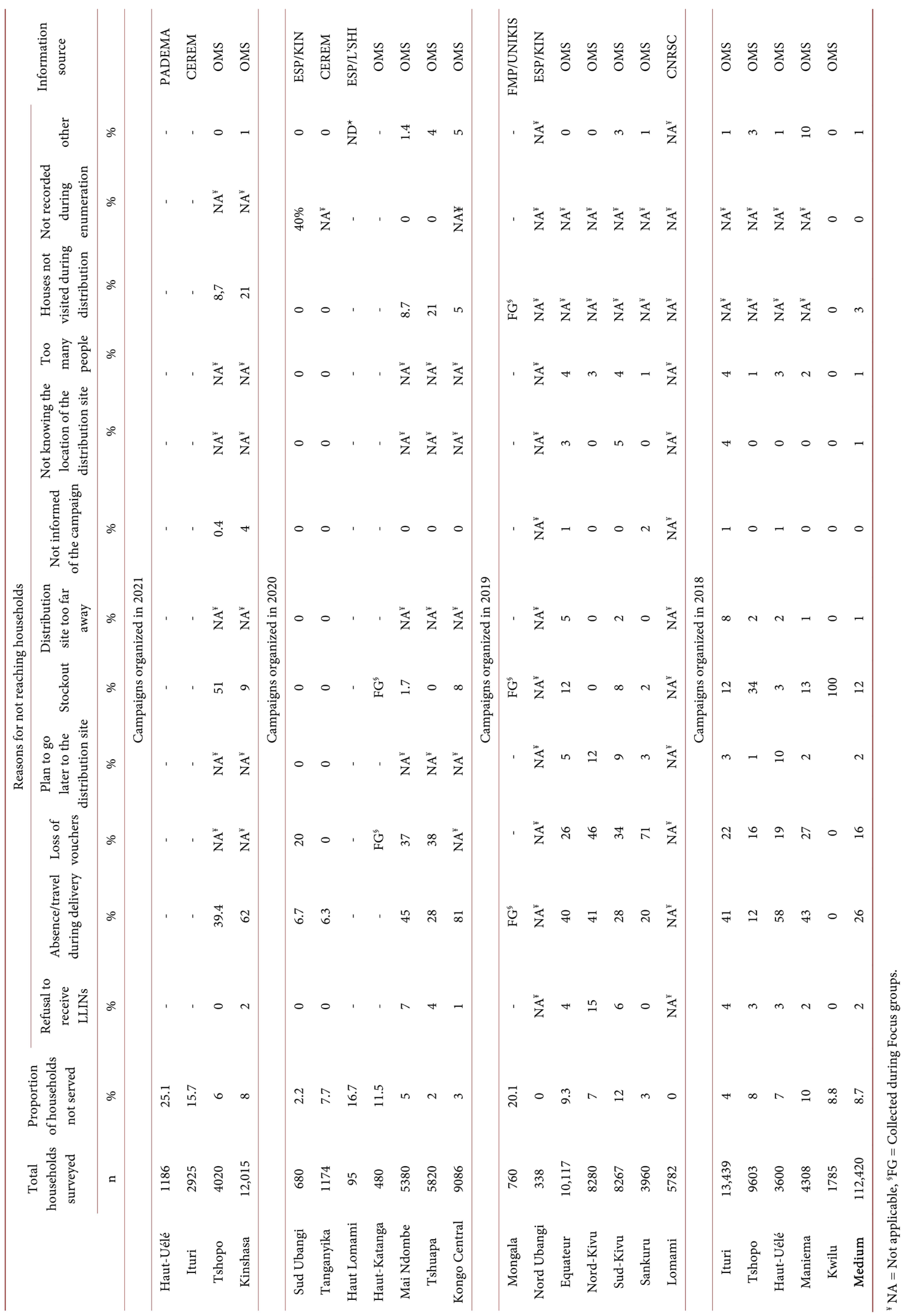


Table 3. The flow of LLINs from forecast to household distribution.

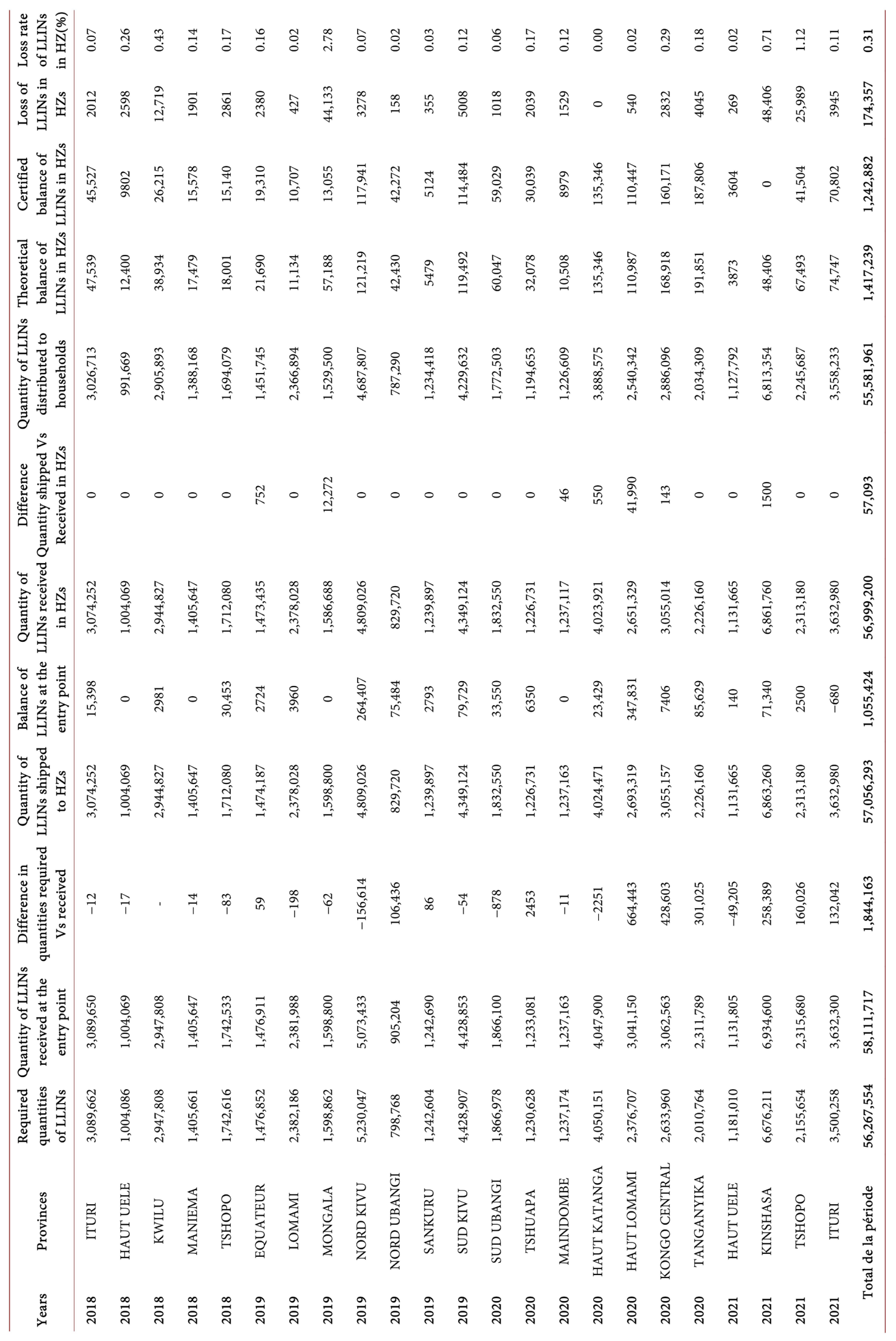


Table 4. Frequency of information sources on LLINs cited by respondents during monitoring.

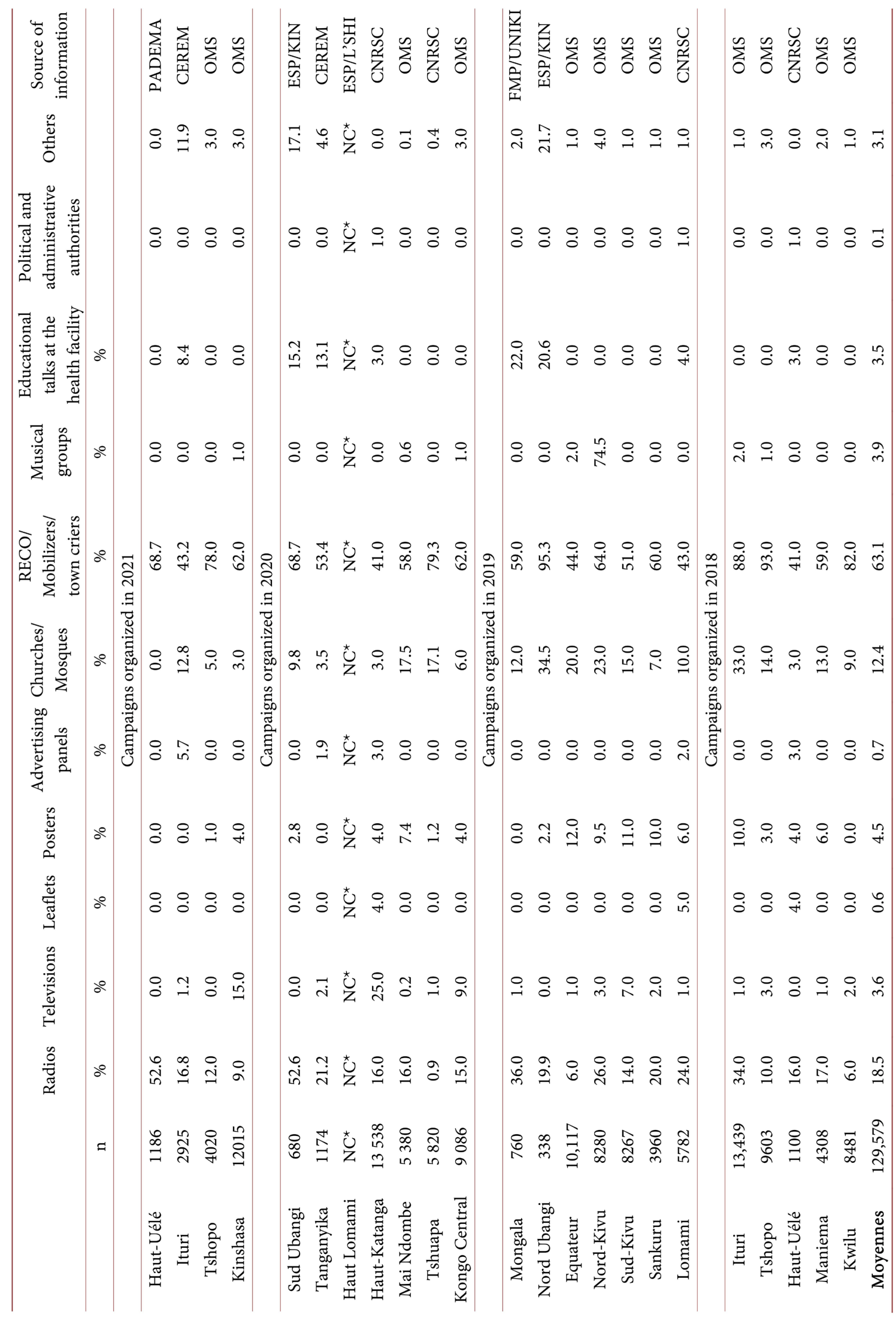




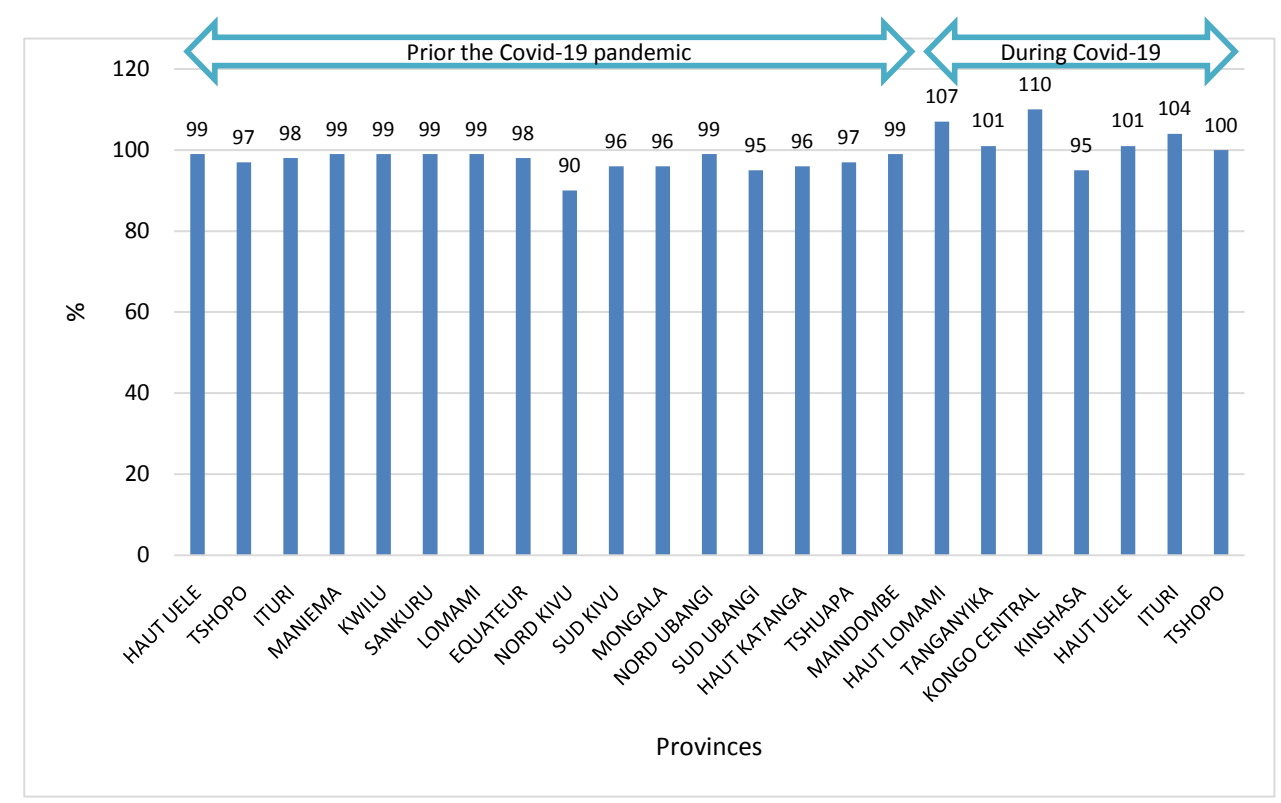

Figure 4. Proportions of LLINs distributed compared to the contractual target.

\section{Discussion}

Between January 2018 and June 2021, 23 free LLIN distribution campaigns were organized in DRC under GFTAM funding with co-financing from AMF resulting in the distribution of 55,581,961 LLINs to $19,048,372$ households at risk of malaria transmission. This corresponds to a LLIN mass distribution for 20 of the 26 provinces of the country in 3 years and a 2nd renewal campaign for 3 of them. Of the six provinces funded by PMI during the same period, two (Kasai and Kasai Oriental) benefited from the planned free distribution, while four (Lualaba, Kwango, Kasai Central and Bas Uélé) were being prepared for distribution in 2021, one of which (Bas-Uélé) has been reallocated to fund by the GFTAM. In a literature review of 14 free LLIN distribution campaigns in East Africa, Sexton et al. noted that programs that financially depend on single donors greatly hamper any possible sustainability [31]. In the case of the DRC, the participation of the AMF in the co-financing of campaigns supported by the GFTAM has been a remarkable contribution to the sustainability of the intervention.

The particularity of the campaigns in the DRC is that they are spread over about 3 years to cover all the 26 provinces of the country, unlike some African countries which distribute throughout the national territory at the same period [32] [33]. Some other countries with heavy malaria burdens and large populations to cover also use a staggered campaign over more than one year [34] [35]. In most cases, this approach, generally dictated by the constraints of gradual mobilization of resources followed by the need to keep a distribution interval of 3 years, has linked the DRC in a configuration of spreading out in 3 years of distributions to cover the country. Additional constraints such as logistical difficulties related to insufficient road infrastructure, armed conflicts, and the COVID-19 
pandemic are added to this, leading to the spread of distributions in some provinces beyond 3 years as is the case for the provinces of Kinshasa, Ituri, HautUélé, and Ituri in the scope of intervention of the GFTAM in the DRC.

On the demographic considerations, DRC LLINs mass distribution campaigns showed discrepancies between the projections made during microplanning and the results (Table 1). Compared to projections, the general trend was to have a more enumerated population ( $+7 \%$ on average) while the number of households was lower ( $-3 \%$ on average). A mass distribution campaign organized in Benin in 2020 showed differences that all went in the same direction: $+9 \%$ of households and $+13 \%$ of the population [32]. In the case of the DRC, this trend probably indicates a propensity to exaggerate the number of people living in the household to benefit more LLINs. Indeed, the NMCP recommends distribution of LLINs according to the size of households at a rate of 1, 2, 3, 4, and 5 LLINs for households of 1 to 2,3 to 4,5 to 6,7 to 8 people, and $\geq 9$ people respectively. As the DRC is between its 3rd and 4th round of LLIN distribution, the population is already aware of the link between household size and the number of LLINs to be received.

These discrepancies make the quantification of LLINs for the countryside more complex, as it depends on a good definition of targets to ensure universal household coverage [30]. Targets are defined based on projection data. Prior to COVID-19, enumeration preceded distribution, leaving a period of adjustment for redeployment of LLINs to the HZs according to their real needs. After the occurrence of COVID-19, the NMCP and its partners had agreed to couple enumeration with distribution to reduce the necessary contacts between the distribution staff and the beneficiaries as recommended by the Alliance for Malaria Prevention (AMP) [36]. As the adjustment window was thus compromised, the NMCP and the partners considered 2 measures to reduce the risks of stock-outs and minimize their impact: 1) an additional order of $5 \%$ of LLINs and 2) the postponement of distribution in at least one HA in each HZ to allow the CT-HZ to deploy its stock to the other HA if necessary and to await the reconciliation of stocks at the end of the distribution in the 1st series of HA and the redeployments between $\mathrm{HA}$ and between $\mathrm{HZ}$ to finalize the distribution. In the complex emergency context such as in Ituri where to the COVID-19 was added an active armed conflict, the number of HA shifted in the city of Bunia which hosted the majority of the displaced populations of the surrounding HZ, the number of HA staggered went up to $3 \mathrm{HA}$ to minimize the risk of stock out.

These provisions made it possible to achieve good performance in the distribution in the context of COVID-19 ( $\geq 95)$ as before COVID-19 (Figure 3). While the administrative data indicated an average coverage of $96 \%$ compared to the projections made at the micro plans, the independent monitoring carried out 2 to 7 days after the visit of the distribution agents showed that $91 \%$ of the households surveyed had been served in LLINs. The difference of $5 \%$ shows the success of the campaigns organized to cover households at malaria risk transmis- 
sion in DRC.

The results of the various monitoring (Table 2) show that, on average, $8.7 \%$ of households surveyed were not served with LLINs, and the reasons for not receiving them were mainly absence from home at the time of distribution and loss of vouchers. Some authors also mentioned travel, household members not being on the enumeration distribution list, stock-outs, the absence or loss of a vouchers, not having an official identity card, and insecurity [35] [37]. After the occurrence of COVID-9, some of the reasons previously mentioned no longer applied in a distribution coupling the enumeration to the distribution, in particular the loss of the voucher. It thus appears that the risk of stock-outs due to an unreliable quantification in the absence of updated enumeration was compensated for by better access to households in a door-to-door approach combining enumeration of household members and immediate delivery of LLINs in the function the number of people living in these households.

Good supply chain management is necessary for the success of campaigns. In the case of the DRC, the supply chain management from orders based on a quantification of needs in accordance with recommendations of the WHO and the AMP, the flexibility of the AMF to add a contingency stock to compensate for possible stock-outs in the context of COVID-19 having made it necessary to skip a preliminary enumeration step before distribution and the logistical follow-up ensuring good traceability of the inputs from the front door to households have made it possible to ensure the distribution of $98 \%$ LLINs targeted for distribution with deviations $\leq 2 \%$ (LLINs received vs. LLINs shipped to $\mathrm{HZs}=1.8 \%$, LLINs shipped to HZs vs. LLINs received at HZs $=0.1 \%$, LLIN loss rate in HZs = $0.31 \%$ ) (Table 3 ). The various deviations included: 1 ) quantities of LLINs reallocated (leftovers) to routine distribution with an option to subsequently recover stocks from routine distribution to campaigns in other provinces, 2) quantities of LLINs (balance after taking microplanning into account) reallocated directly to campaigns in other provinces, 3) marginally, there were losses due to shipwrecks or losses at warehouses or during transport. These losses were subject to restitution either by insurers or by deduction from the payments of service providers (transporters or warehouse owners).

Multi-channel behavior change communication campaigns are effective in increasing the culture of net attachment and use, especially among vulnerable groups [38]. As was the case in the DRC (Table 4), proximity communication using different communication channels (radio, television, leaflets, $\mathrm{CHW}$, etc.) has been taken up by several authors. CHWs, churches and community leaders have been found to be the main sources of information for households in several studies of mass distribution experiences in Africa [35] [38]. In Tanzania, Reggli et al. reported radio as the primary source of information in an approach that used community outreach radio stations more than national radio and television stations [39]. The geographic coverage of radio stations varying from one country to another and within the same country from one province to another could ex- 
plain this variability.

One of the limitations of the evaluation of the campaigns in the DRC is the lack of standardization of independent monitoring, which results in some missing data and a variability of methodological approaches that is detrimental to the strict comparison of results. In addition, the digitization of the management of free LLIN distribution campaigns only takes into account enumeration and distribution data. A roadmap was drawn up by the NMCP and its partners to add modules relating to the management of training, supervision, and input tracking.

\section{Conclusion}

The DRC organized an LLIN replacement cycle in the particular context of the COVID-19 pandemic and armed conflicts in the eastern provinces. Despite the many challenges of its implementation, the LLIN campaigns were successfully implemented and contributed to increasing household coverage and population access to LLINs. Good planning, effective coordination of stakeholders, and the revision of campaign implementation modalities in line with COVID-19 were factors of the success of this campaign. An effort to respect household LLIN renewal schedules, coupled with good routine distribution, is necessary to maintain the gains and hope for an impact in terms of reducing malaria morbidity and mortality.

\section{Acknowledgements}

The data used in this article are programmatic data from the LLIN mass distribution project co-funded by GFTAM and AMF implemented by SANRU Asbl, to whom we are grateful.

\section{Conflicts of Interest}

The authors declare no conflicts of interest regarding the publication of this paper.

\section{References}

[1] WHO (2015) Global Technical Strategy for Malaria 2016-2030. World Health Organization, Geneva.

[2] RBM (2015) Action et Investissement pour vaincre le paludisme 2016-2030. Roll Back Malaria Partenariat pour en finir avec le paludisme, Genève.

[3] WHO (2016) World Malaria Report 2016. World Health Organization, Geneva.

[4] WHO (2020) World Malaria Report 2020. World Health Organization, Geneva.

[5] WHO (2019) High Burden to High Impact: A Targeted Malaria Response. World Health Organization, Geneva.

https://apps.who.int/iris/bitstream/handle/10665/275868/WHO-CDS-GMP-2018.25 -eng.pdf?ua $=1$

[6] Programme national de lutte contre le paludisme (2020) Plan stratégique national de lutte contre le paludisme 2020-2023. PNLP, Ministère de la santé, RDC. 
[7] Pryce, J., Richardson, M. and Lengeler, C. (2018) Insecticide-Treated Nets for Preventing Malaria. Cochrane Database of Systematic Reviews, No. 11. CD000363. https://doi.org/10.1002/14651858.CD000363.pub3

[8] Lim, S., Fullman, N., Stokes, A., Ravishankar, N., Masiye, F., Murray, C.L., et al. (2011) Net Benefits: A Multicountry Analysis of Observational Data Examining Associations between Insecticide-Treated Mosquito Nets and Health Outcomes. PLOS Medicine, 8, e1001091. https://doi.org/10.1371/journal.pmed.1001091

[9] Shargie, E.B., Ngondi, J., Graves, P.M., Getachew, A., Hwang, J., Gebre, T., et al. (2010) Rapid Increase in Ownership and Use of Long-Lasting Insecticidal Nets and Decrease in Prevalence of Malaria in Three Regional States of Ethiopia (2006-2007). Journal of Tropical Medicine, 2010, Article ID: 750978. https://doi.org/10.1155/2010/750978

[10] Otten, M., Aregawi, M., Were, W., Karema, C., Medin, A., Bekele, W., et al. (2009) Initial Evidence of Reduction of Malaria Cases and Deaths in Rwanda and Ethiopia Due to Rapid Scale-Up of Malaria Prevention and Treatment. Malaria Journal, 8, Article No. 14. https://doi.org/10.1186/1475-2875-8-14

[11] WHO (2020) The Potential Impact of Health Service Disruptions on the Burden of Malaria: A Modelling Analysis for Countries in Sub-Saharan Africa. https://www.who.int/publications/i/item/9789240004641

[12] Hogan, A.B., Jewell, B.L., Sherrard-Smith, E., Vesga, J.F., Watson, O.J., Whittaker, C., et al. (2020) Potential Impact of the COVID-19 Pandemic on HIV, Tuberculosis, and Malaria in Low-Income and Middle-Income Countries: A Modelling Study. The Lancet Global Health, 8, e1132-e1141. https://doi.org/10.1016/S2214-109X(20)30288-6

[13] Rogerson, S.J., Beeson, J.G., Laman, M., Poespoprodjo, J.R., William, T., Simpson, J.A., et al. (2020) Identifying and Combating the Impacts of COVID-19 on Malaria. BMC Medicine, 18, Article No. 239. https://doi.org/10.1186/s12916-020-01710-x

[14] Ministère du plan, Macro International Inc. (2008) Enquête Démographique et de Santé (EDS-RDC) 2007. Calverton.

[15] Ministère du Plan et Suivi de la Mise en ouvre de la Révolution de la Modernité (MPSMRM), Ministère de la Santé Publique (MSP), ICF International (2014) Enquête Démographique et de Santé en République Démocratique du Congo 2013-2014. MPSMRM, MSP and ICF International, Rockville.

[16] Levitz, L., Janko, M., Mwandaglirwa, K., Thwai, K.L., Likwela, J.L., Tshefu, A.K., et al. (2018) Effect of Individual and Community-Level Net Usage on Malaria Prevalence among Under-Fives in the Democratic Republic of Congo. Malaria Journal, 17, Article No. 39. https://doi.org/10.1186/s12936-018-2183-y

[17] Dolan, C.B., Yishay, A.B., Grépin, K.A., Tanner, J.C., Kimmel, A.D., Wheeler, D.C., et al. (2019) The Impact of an Insecticide Treated Bednet Campaign on All-Cause Child Mortality: A Geospatial Impact Evaluation from the Democratic Republic of Congo. PLoS ONE, 14, e021890. https://doi.org/10.1371/journal.pone.0212890

[18] Karemere, H., Nana, I.G., Adrada, A., Kakesa, O., Sompwe, E.M., Losimba, J.L., et al. (2021) Associating the Scale-Up of Insecticide-Treated Nets and Use with the Decline in All-Cause Child Mortality in the Democratic Republic of Congo from 2005 to 2014. Malaria Journal, 20, Article No. 241.

https://doi.org/10.1186/s12936-021-03771-6

[19] Global Malaria Programme, WHO (2017) Revised Recommendations for Achieving Universal Coverage with Long-Lasting Insecticidal Nets in Malaria Control. World Health Organization, Geneva. 
https://www.who.int/malaria/mpac/mpac-oct2017-erg-universal-coverage-session9presentation.pdf?ua $=1$

[20] Programme national de lutte contre le paludisme (2017) Rapport d'activités 2016. PNLP, Ministère de la santé, RDC.

[21] Programme national de lutte contre le paludisme (2018) Rapport d'activités 2017. PNLP, Ministère de la santé, RDC.

[22] Programme national de lutte contre le paludisme (2019) Rapport d'activités 2018. PNLP, Ministère de la santé, RDC.

[23] Programme national de lutte contre le paludisme (2016) PSN 2016-2020. PNLP, Ministère de la santé, RDC.

[24] Brazeau, N.F., Whitesell, A.N., Doctor, S.M., Keeler, C., Mwandagalirwa, M.K., Tshefu, A.K., et al. (2018) Plasmodium vivax Infections in Duffy-Negative Individuals in the Democratic Republic of the Congo. American Journal of Tropical Medicine and Hygiene, 99, 1128-1133. https://doi.org/10.4269/ajtmh.18-0277

[25] Brazeau, N.F., Mitchell, C.L., Morgan, A.P., Deutsch-Feldman, M., Watson, O.J., Thwai, K.L., et al. (2021) The Epidemiology of Plasmodium vivax among Adults in the Democratic Republic of the Congo. Nature Communications, 12, Article No. 4169. https://doi.org/10.1038/s41467-021-24216-3

[26] Wat'senga, F., Manzambi, E.Z., Lunkula, A., Mulumbu, R., Mampangulu, T., Lobo, N., et al. (2018) Nationwide Insecticide Resistance Status and Biting Behaviour of Malaria Vector Species in the Democratic Republic of Congo. Malaria Journal, 17, Article No. 129. https://doi.org/10.1186/s12936-018-2285-6

[27] Programme national de lutte contre le paludisme (2017) Manuel de planification et de mise en ouvre des campagnes de distribution de masse des moustiquaires imprégnées à longue durée. PNLP, Ministère de la santé, RDC.

[28] Programme national de lutte contre le paludisme (2020) Manuel de planification et de mise en œuvre des campagnes de distribution de masse des moustiquaires imprégnées à longue durée dans le contexte de pandémie de COVID-19. PNLP, Ministère de la santé, RDC.

[29] Ministère du Plan, Institut National de la Statistique (2014) Annuaire statistique 2014.

[30] Alliance pour la prévention du paludisme (2012) Boîte à outils pour les campagnes de distribution massive visant à augmenter la couverture et l'utilisation de moustiquaires imprégnées d'insecticide longue durée. Seconde édition.

[31] Sexton, A.R. (2011) Best Practices for an Insecticide-Treated Bed Net Distribution Programme in Sub-Saharan Eastern Africa. Malaria Journal, 10, Article No. 157. https://doi.org/10.1186/1475-2875-10-157

[32] Aïkpon, R., Afoukou, C., Hounpkatin, B., Eclou, D.D., Cyaka, Y. and Egwu, E. (2020) Digitalized Mass Distribution Campaign of Insecticide-Treated Nets (ITNs) in the Particular Context of COVID-19 Pandemic in Benin: Challenges and Lessons Learned. Malaria Journal, 19, Article No. 431. https://doi.org/10.1186/s12936-020-03508-x

[33] Bennett, A., Smith, S.J., Yambasu, S., Jambai, A., Alemu, W., Kabano, A., et al. (2012) Household Possession and Use of Insecticide-Treated Mosquito Nets in Sierra Leone 6 Months after a National Mass-Distribution Campaign. PLoS ONE, 7, e37927. https://doi.org/10.1371/journal.pone.0037927

[34] Saleh, J.E.A., Uchenna, A.A., Saddiq, A., Wondimagegnehu, A., Mpazanje, R. and Audu, B.M. (2018) Lots Quality Assurance Survey (LQAS) as a Strategy to Achiev- 
ing Quality LLIN Campaigns: The Nigerian Experience. Open Access Library Journal, 5, e4484. https://doi.org/10.4236/oalib.1104484

[35] Finlay, A.M., Butts, J., Ranaivoharimina, H., Cotte, A.H., Ramarosandratana, B., Rabarijaona, H., Tuseo, L., Chang, M. and Vanden Eng, J. (2017) Free Mass Distribution of Long Lasting Insecticidal Nets Lead to High Levels of LLIN Access and Use in Madagascar, 2010: A Cross-Sectional Observational Study. PLoS ONE, 12, e0183936. https://doi.org/10.1371/journal.pone.0183936

[36] Alliance pour la prévention du paludisme (2020) Orientations essentielles pour la distribution de moustiquaires imprégnées d'insecticide dans le contexte de la transmission du COVID-19. V.2 juillet.

[37] Linn, S.Y., Maung, T.M., Tripathy, J.P., Shewade, H.D., Oo, S.M., Linn, Z. and Thi, A. (2019) Barriers in Distribution, Ownership and Utilization of Insecticide-Treated Mosquito Nets among Migrant Population in Myanmar, 2016: A Mixed Methods Study. Malaria Journal, 18, Article No. 172.

https://doi.org/10.1186/s12936-019-2800-4

[38] Masaninga, F., Mukumbuta, N., Ndhlovu, K., Hamainza, B., Wamulume, P., Chanda, E., Banda, J., Mwanza-Ingwe, M., Miller, J.M., Ameneshewa, B., Mnzava, A. and Kawesha-Chizema, E. (2018) Insecticide-Treated Nets Mass Distribution Campaign: Benefits and Lessons in Zambia. Malaria Journal, 17, Article No. 173. https://doi.org/10.1186/s12936-018-2314-5

[39] Renggli, S., Mandike, R., Kramer, K., Patrick, F., Brown, N.J., McElroy, P.D., Rimisho, W., Msengwa, A., Mnzava, A., Nathan, R., Mtung'e, R., Mgullo, R., Lweikiza, J. and Lengeler, C. (2013) Design, Implementation and Evaluation of a National Campaign to Deliver 18 Million Free Long-Lasting Insecticidal Nets to Uncovered Sleeping Spaces in Tanzania. Malaria Journal, 12, Article No. 85.

https://doi.org/10.1186/1475-2875-12-85 